\title{
Conhecimento ecológico tradicional sobre fruteiras para pesca na Comunidade de Estirão Comprido, Barão de Melgaço - Pantanal Matogrossense
}

\author{
Fernando Ferreira de Morais ${ }^{1,3}$ \& Carolina Joana da Silva ${ }^{1,2}$ \\ ${ }^{1}$ Programa de Pós-graduação em Ciências Biológicas (Biologia Vegetal), \\ Universidade Estadual Paulista "Júlio de Mesquita Filho" - UNESP \\ Av. 24 A, 1515, CEP 13506-900, Bela Vista, Rio Claro, SP, Brasil \\ ${ }^{2}$ Centro de Pesquisa em Limnologia, Biodiversidade e Etnobiologia do Pantanal, \\ Universidade do Estado de Mato Grosso - UNEMAT, \\ Av. Tancredo Neves, 1060, CEP 78200-000, Cáceres, MT, Brasil \\ ${ }^{3}$ Autor para correspondência: Fernando Ferreira de Morais, e-mail: moraisff@hotmail.com
}

MORAIS, F.F. \& SILVA, C.J. Traditional ecological knowledge of fruit trees used for fishery at Estirão Comprido Community, Barão de Melgaço - Panatanal Matogrossense. Biota Neotrop. 10(3): http://www. biotaneotropica.org.br/v10n3/en/abstract?article+bn03510032010.

\begin{abstract}
This study deals with traditional ecological knowledge on fruit trees at Estirão Comprido community, located on the banks of Cuiabá River, in Barão de Melgaço, Pantanal Matogrossense. Twenty fishermen were interviewed, using free lists, which were evaluated by Smith's Salience Index and cultural consensus analysis, using the software ANTHROPAC 4.0. Results indicated that traditional ecological knowledge on fruit trees involves 30 species distributed in 21 families. Smith's Salience Index showed four ruptures on the rank and frequency of answers; the first rupture included one species, the second eight species, the third four species and fourth 17 species. The cultural consensus of fishermen on such traditional knowledge concentrated on nine fruit tree species: "parada", "coquinho", "marmelada", "roncador", "sardinha", "timbó", "acaiá", "jenipava" and "taiuiá", verified by the relationship between factors, where the first factor was approximately nine-fold higher than the second one, pseudo-reliability $=0.951$. This study indicated that Pantanal community presents knowledge about fruit trees, the base of the food chain for commercially important fish.
\end{abstract}

Keywords: Pantanal, traditional community, fruit plants, fishing.

MORAIS, F.F. \& SILVA, C.J. Conhecimento ecológico tradicional sobre fruteiras para pesca na Comunidade de Estirão Comprido, Barão de Melgaço - Pantanal Matogrossense. Biota Neotrop. 10(3): http://www. biotaneotropica.org.br/v10n3/pt/abstract?article+bn03510032010.

Resumo: Esta pesquisa aborda o conhecimento ecológico tradicional de fruteiras na comunidade de Estirão Comprido, localizada às margens do Rio Cuiabá, Barão de Melgaço, Pantanal Matogrossense. Foram entrevistados 20 pescadores, utilizando-se a técnica de listagem livre, a qual foi analisada pelo índice de saliência de Smith e análise de consenso cultural, com o programa ANTHROPAC 4.0. Os resultados mostraram que o conhecimento ecológico tradicional sobre fruteiras foi de 30 espécies distribuídas em 21 famílias de plantas. A análise de índice de saliência de Smith mostrou quatro rupturas quanto à ordenação e frequência de respostas, sendo que a primeira contemplou uma espécie, a segunda oito espécies, a terceira quatro espécies e a quarta ruptura 17 espécies. O consenso cultural dos pescadores sobre o conhecimento ecológico tradicional concentrou-se em nove espécies de fruteiras: parada, coquinho, marmelada, roncador, sardinha, timbó, acaiá, jenipava e taiuiá, verificado pela relação entre fatores, onde o primeiro fator foi aproximadamente nove vezes maior que o segundo fator com probabilidade $=0,951$. O estudo evidenciou que esta comunidade pantaneira apresenta um conhecimento sobre as fruteiras que são a base da cadeia alimentar das espécies de peixes de valor comercial.

Palavras-chave: Pantanal, comunidade tradicional, plantas frutíferas, pesca. 


\section{Introdução}

As comunidades tradicionais no Pantanal mantêm uma relação estreita com a dinâmica das águas, adaptando suas estratégias de vida associadas aos ciclos naturais que regem este bioma, o que lhes possibilita aplicar seus conhecimentos ecológicos tradicionais no manejo dos recursos necessários à continuidade cultural (Da Silva \& Silva 1995).

Neste sentido, o conhecimento ecológico tradicional é característico das Comunidades Tradicionais e de acordo com Barreto Filho (2006), a noção de "população tradicional" expressa um conjunto de valores culturais coletivos relativos ao meio ambiente.

Dentre as abordagens para estudos com comunidades tradicionais, podemos destacar a etnobiologia que, de acordo com Ellen (2006), é considerada o estudo de como as pessoas de quaisquer tradições culturais interpretam, utilizam e em geral gerenciam seus conhecimentos sobre os domínios da experiência ambiental, que englobam os organismos vivos e cujo estudo cientifico é delimitado pela botânica, zoologia, ecologia entre outras.

Neste contexto, o Conhecimento Ecológico Tradicional (CET) é entendido como um corpo cumulativo de conhecimento, práticas e crenças, sobre as relações entre os seres vivos e o meio ambiente, que evolui e é repassado por gerações através da cultura (Berkes et al. 1998). O CET também é traduzido no contato direto com os recursos naturais, a observação diária desses recursos e a dependência econômica de recursos aquáticos e da vegetação que representam relações ecológicas em seu sentido estrito (Begossi 2004). Nessa abordagem, Fraser et al. (2006) destacam que o CET pode contribuir para proteção de habitats e espécies culturalmente importantes e ameaçadas de extinção, além de colaborar para o planejamento e conservação da biodiversidade, mantendo a diversidade ecológica e genética e os processos evolutivos.

Vários são os estudos sobre o uso e manejo de recursos naturais no Pantanal e na Bacia do Alto Paraguai (Campos Filho 2002, Bertsch et al. 2006, Pasa 2007, Guarim Neto \& Carniello 2007, Bortolotto \& Guarim-Neto 2005), alguns enfocando a descrição e manejo da pecuária (Da Silva \& Silva 1995, Campos Filho 2002), da pesca (Da Silva \& Silva 1995) e outros, as diferentes formas de uso e manejo dos recursos vegetais (Anjos Silva \& Da Silva 2000, Schwenk \& Da Silva 1999, Pasa et al. 2005, Guarim Neto 2006, Galdino \& Da Silva 2007).

Estudos sobre recursos vegetais, como frutos e sementes na Amazônia, mostram a importância destes na alimentação de peixes que em muitos casos efetuam a dispersão de várias plantas (Gottsberger 1978, Goulding et al. 1988, Kubitzki \& Ziburski 1994, Maia 2001). Espécies de peixes como Colossoma macropomum Cuvier 1818 e Lithodoras dorsalis Valenciennes 1840, alimentam-se exclusivamente de frutas e sementes durante o período de submersão das florestas inundáveis na Amazônia (Kubitzki \& Ziburski 1994).

No Pantanal, algumas espécies da floresta inundável como tucum (Arecaceae), a figueira (Moraceae), o acaiá (Anacardiaceae), o roncador (Melastomataceae), jenipava (Rubiaceae), tarumã (Verbenaceae) são consideradas importantes recursos vegetais (Anjos Silva \& Da Silva 2000), uma vez que diversas espécies de peixes apresentam guildas relacionadas a essas plantas (Da Silva \& Silva 1995), como por exemplo Brycon hilarii (Zuntini et al. 2004, Reys et al. 2009), Piaractus mesopotamicus (Ferreira et al. 2000, Galetti et al. 2007). Para Hanazaki (2004), cada vez mais são necessários estudos direcionados às condições que refletem ou não a conservação, bem como às práticas de manejo de recursos vegetais utilizadas pelas populações locais.

Com base nestas considerações, este estudo teve como objetivo investigar o conhecimento ecológico tradicional sobre fruteiras conhecidas para pesca por pescadores da Comunidade Tradicional de Estirão Comprido no Pantanal Matogrossense.

\section{Material e Métodos}

\section{1. Área de estudo}

O Pantanal encontra-se no centro da América do Sul, compreendendo parte da Bolívia, Paraguai e com maior extensão no Brasil, nos Estados de Mato Grosso e Mato Grosso do Sul. É uma depressão sazonalmente alagável, totalmente contida na bacia de drenagem do Alto Paraguai e compreende aproximadamente $140.000 \mathrm{~km}^{2}$ (Brasil 1982). O clima da bacia do Rio Cuiabá é do tipo (AW-Köppen), com duas estações bem definidas, compreendendo a seca (dos meses de maio a setembro) e a chuvosa (dos meses de novembro a abril). A temperatura média anual é $26^{\circ} \mathrm{C}$, com a maior média de temperatura em outubro $\left(27,6{ }^{\circ} \mathrm{C}\right.$ ) e a menor em julho com $23{ }^{\circ} \mathrm{C}$ (Figueiredo \& Da Silva 1999). No Pantanal, a biodiversidade varia entre e dentro das manchas que formam um mosaico de habitat não inundáveis e sazonalmente inundáveis e permanentemente aquáticos (Da Silva et al. 2001).

O estudo foi realizado na zona rural do município de Barão de Melgaço, na Comunidade de Estirão Comprido, localizada as margens do Rio Cuiabá ( $16^{\circ} 16^{\prime} 50^{\prime \prime} \mathrm{S}$ e $55^{\circ} 58^{\prime} 58^{\prime}$ ' W) distante $15 \mathrm{~km}$ da sede do município no Pantanal Matogrossense - Mato Grosso. As principais atividades econômicas do município são: pesca, agricultura de subsistência, pecuária de gado de corte, retirada de isca viva para pesca e turismo de pesca. Barão de Melgaço possui uma área de $11,183 \mathrm{~km}^{2}$ e 7,619 habitantes (IBGE 2002) e, destes, 296 residem na Comunidade de Estirão Comprido.

\section{Métodos}

Nesta pesquisa, foram entrevistados $23 \%$ (20) dos pescadores da comunidade, amostrados pelo método de bola de neve (Bernard 2002) para obtenção da listagem livre referente às fruteiras conhecidas para pescar. Para Borgatti (1996a) a listagem livre é uma ferramenta eficiente para indicar quais itens pertencem ao domínio cultural, e refere-se a um grupo de palavras organizadas, conceitos ou sentenças, nesta pesquisa, fruteiras, todas com mesmo nível de contraste, conjuntamente na mesma esfera conceitual (Weller \& Romney 1988).

Os dados obtidos foram analisados pelo índice de saliência de Smith, consenso cultural, escalonamento multidimensional e técnica de empilhamento "Pilesort" (Borgatti 1996b, Bernard 2002). O índice de Smith é uma medida da saliência de cada item da listagem livre, que vai de 0 a 1 e se baseia nos maiores valores de frequência absoluta e maior coincidência de posição de citação dos itens da listagem livre entre os informantes, promovendo assim um ordenamento dos itens da listagem, o que permite encontrar possíveis 'quebras' ou rupturas entre um item e outro relacionado na listagem livre. Isto ocorre devido a alguns itens da listagem serem mencionados por muitos informantes e outros por poucos ou por apenas um informante (Puri 2001). Neste sentido, as rupturas são agrupamentos de itens do domínio cultural estabelecidos pelo informante, de acordo com sua importância cultural, assim itens citados com frequência e ordem de citações semelhantes tendem a formar grupos, estabelecendo assim rupturas (Morais et al. 2009).

A análise de escalonamento multidimensional oferece uma representação visual dos padrões de similaridade ou dissimilaridade entre o grupo de objetos estudados e com a técnica de empilhamento, podem-se testar hipóteses para interpretação dos dados por meio da similaridade e dissimilaridade entre os itens da listagem livre (Borgatti 1996b). A análise de consenso cultural, obtida a partir dos dados da listagem livre, foi realizada com intuito de atender os objetivos preconizados por Caulkins \& Hyatt (1999) para utilização desses conceitos: 1) o grau de concordância entre os informantes sobre o domínio do conhecimento; 2) a informação "culturalmente correta" é definida pelo informante através do seu conceito cultural e não pela ótica científica (Borgatti 1996b), sobre o domínio cultural 
e as respostas comuns dos informantes; 3) uma contagem para cada informante representando o domínio do conhecimento. Na análise de consenso cultural, o primeiro fator (itens do consenso cultural) deve ser no mínimo três vezes maior que o segundo fator (demais itens da listagem livre) para que possa ser atribuído consenso entre os informantes (Borgatti 1996b).

Com base nas interpretações dos dados de similaridade e dissimilaridade obtidos após análise da listagem livre, utilizou-se, com onze pescadores, a técnica de empilhamento (Borgatti 1996b) para testar a hipótese de que existem fruteiras específicas utilizadas pelos pescadores para capturar peixes de maior valor comercial. Os resultados obtidos no empilhamento foram analisados por meio da análise de consenso cultural e da análise de escalonamento multidimensional (Borgatti 1996b, Bernard 2002).

As plantas indicadas na listagem livre foram coletadas, identificadas e estão depositadas no herbário da Universidade Federal de Mato Grosso - UFMT.

\section{Resultados}

Os moradores da comunidade apresentam alto grau de parentesco, são oriundos de Barão de Melgaço e diferentes comunidades pantaneiras deste município ou muito próximas deste (Morais et al. 2009) que possuem características socioeconômicas e culturais similares (Da Silva \& Silva 1995), fato que favorece o consenso no domínio cultural observado nesse estudo. Foram entrevistados somente homens e a idade variou de 26 a 66 anos, com uma média de 47 anos. Todos os pescadores entrevistados complementam sua renda com atividades extra pesca, sendo que 19 disseram ser também lavradores e um piloteiro de embarcações em pousadas da região. O baixo nível escolar entre os entrevistados também é comum, sendo que a maioria sequer chegou ao ensino fundamental (Galdino \& Da Silva 2007, Morais et al. 2009).

\section{Fruteiras para pesca na comunidade de Estirão Comprido}

$\mathrm{Na}$ análise de listagem livre sobre o conhecimento ecológico tradicional das fruteiras para pescar, foi possível verificar que o domínio cultural concentra-se em 30 espécies pertencentes a 21 famílias (Tabela 1). A análise do índice de saliência de Smith mostrou quatro rupturas ou saliências no domínio cultural sobre plantas frutíferas conhecidas para pescar. A primeira ruptura concentra apenas uma espécie, a parada (Pouteria glomerata (Miq.) Radlk); a segunda ruptura contempla oito espécies, roncador (Mouriri guianensis Aubl.), marmelada (Alibertia sp.), coquinho (Bactris glaucescens Drude), sardinheira (Banara arguta Briq.), timbó (Paullinia sp.), jenipava (Genipa americana L.), taiuiá (Cayaponia podantha Cogn.) e acaiá (Spondias lutea L.). Na terceira ruptura encontram-se quatro espécies: canjiqueira (Byrsonima orbigniana A. Juss), coquinho preto (Bactris sp. 1), coquinho amarelo (Bactris sp. 2) e sarã de leite (Sapium obovatum Kl.), e na quarta e última ruptura encontram-se 17 espécies (Tabela 1).

A análise de consenso cultural das fruteiras mostrou consenso entre os pescadores (o primeiro fator 10,215 é maior que o segundo fator 1,017, com probabilidade $=0,950$ ) em nove espécies de fruteiras: parada, roncador, marmelada, coquinho, sardinheira, timbó, jenipava, taiuiá e acaiá, e a estimativa do conhecimento dos pescadores em relação ao domínio cultural de fruteiras conhecidas para pescar, apresentou uma média de 0,70 e um desvio padrão de 0,14 (Tabela 2), significando pouca variação entre o conhecimento dos informantes.

O diagrama de escalonamento multidimensional correspondente ao agrupamento dos pescadores, pela concordância das respostas sobre as fruteiras conhecidas na utilização da pesca, mostrou a formação de dois grupos, um com 19 pescadores e outro com somente um pescador (Figura 1). Essa diferença pode ser devido ao pescador, que esta mais distante do centro do diagrama de escalonamento multidimensional, indicar fruteiras na listagem livre que não fazem parte do consenso cultural dos pescadores da comunidade, como é o caso da acuri (Scheelea phalerata), bocaiúva (Acrocomia aculeata) e manga (Mangifera indica). Além disso, o pescador utiliza técnicas diferenciadas no preparo de iscas para pescar com o acuri e bocaiúva, que consiste no cozimento do fruto para retirada do mesocarpo e posterior mistura com farinha de trigo. Muito embora não haja consenso nas indicações das espécies por este pescador, observou-se que sua origem é a mesma dos demais pescadores da comunidade, e provavelmente as técnicas de manejo e o conhecimento sobre essas espécies provêm de experiências pessoais ou troca de experiências com pessoas externas à comunidade.

Em relação ao diagrama de escalonamento multidimensional do agrupamento das plantas frutíferas, de acordo com as respostas dos pescadores, houve a separação em dois grupos, um formado por nove espécies e outro por 21 espécies. O primeiro grupo, formado pelas espécies do consenso cultural, contempla as espécies da primeira e segunda rupturas da listagem livre: parada, sardinheira, timbó, roncador, coquinho, marmelada, taiuiá, jenipava, acaiá. O segundo grupo é formado por espécies que estão fora do consenso cultural e são pertencentes à terceira e quarta rupturas da listagem livre: canjiqueira, cabaça brava, goiaba, coquinho preto, figueira, pimenteira, coquinho amarelo, cachuá, sarã de espinho, sarã de leite, paineira, acuri, goiaba brava, cabaça grande, carandá, juá, pateiro, caneleira, bocaiúva, ingá de botoado e manga (Figura 2).

A análise de consenso cultural pela técnica de empilhamento para classificar o CET das fruteiras pelo valor de mercado do pescado indicou existir consenso entre os pescadores (o $1^{\circ}$ fator 4,525 é maior que o $2^{\circ}$ fator 0,446 , e a probabilidade do domínio cultural caracterizar um consenso é de 0,873 ) e a estimativa do conhecimento dos pescadores, referente ao empilhamento das fruteiras pelo valor de mercado do pescado, apresentou uma média de 0,62 e um desvio padrão de 0,15 (Tabela 3). Aqui também verificamos baixa variação no conhecimento dos pescadores com relação à escolha da fruteira para capturar peixes de maior valor comercial.

O diagrama de escalonamento multidimensional referente ao agrupamento dos pescadores, de acordo com suas respostas no empilhamento das fruteiras em relação ao valor de mercado do pescado, evidenciou a formação de um grupo com 10 pescadores e outro com um pescador (Figura 3), observamos nos resultados uma grande proximidade entre as respostas dos entrevistados, devido a isto, houve a sobreposição dos mesmos na Figura 3. A análise de escalonamento multidimensional mostrou que os pescadores da Comunidade de Estirão Comprido classificam as fruteiras de acordo com a potencialidade de capturar peixes de maior valor comercial, respectivamente pacu (Piaractus mesopotamicus), piraputanga (Brycon sp.) e pacu-peva (Metynnis sp.)

O diagrama de escalonamento multidimensional referente ao agrupamento das fruteiras no empilhamento para capturar peixes de maior valor de mercado evidenciou a formação de dois grupos, o primeiro com 10 fruteiras; neste, encontram-se as espécies conhecidas como as mais apreciadas para capturar peixes de maior valor comercial. Os locais onde estas espécies crescem são valorizados como habitat de alta produção e por isso servem como local de ceva natural, além de suas frutas serem consideradas eficientes na captura de determinadas espécies de peixes. No segundo grupo estão as outras 20 espécies, as quais são conhecidas para pescar peixes de pequeno porte, mas sua principal função é na alimentação dos peixes em ceva natural (Figura 4). 
Tabela 1. Análise da Listagem Livre das fruteiras conhecidas para pescar pelos pescadores da Comunidade de Estirão Comprido; Índice de Smith.

Table 1. Analysis of the fruit tree free list known by fishermen for fishing in the Community of Estirão Comprido; Smith's index.

\begin{tabular}{|c|c|c|c|c|c|c|}
\hline Itens & Nome popular & Família & $\begin{array}{c}\text { Espécies } \\
\end{array}$ & Frequência $(\%)$ & Ranque & Índice de Smith \\
\hline 1 & Parada & Sapotaceae & Pouteria glomerata (Miq.) Radlk & 100 & 1.950 & 0,895 \\
\hline 2 & Roncador & Melastomataceae & Mouriri guianensis Aubl. & 80 & 4.500 & $\mathbf{0 , 5 0 1}$ \\
\hline 3 & Marmelada & Rubiaceae & Alibertia sp. & 75 & 4.533 & 0,479 \\
\hline 4 & Coquinho & Arecaceae & Bactris glaucescens Drude & 80 & 5.250 & 0,466 \\
\hline 5 & Sardinheira & Flacourtiaceae & Banara arguta Briq. & 90 & 6.111 & 0,431 \\
\hline 6 & Timbó & Sapindaceae & Paullinia sp. & 80 & 5.875 & 0,401 \\
\hline 7 & Jenipava & Rubiaceae & Genipa americana $\mathrm{L}$. & 70 & 5.643 & 0,396 \\
\hline 8 & Taiuiá & Cucurbitaceae & Cayaponia podantha Cogn. & 70 & 5.500 & 0,381 \\
\hline 9 & Acaiá & Anacardiaceae & Spondias lutea $\mathrm{L}$. & 60 & 6.833 & 0,272 \\
\hline 10 & Canjiqueira & Malpighiaceae & Byrsonima orbignyana A. Juss & 30 & 7.833 & 0,116 \\
\hline 11 & Coquinho Preto & Arecaceae & Bactris sp 1. & 20 & 5.250 & 0,109 \\
\hline 12 & $\begin{array}{l}\text { Coquinho } \\
\text { Amarelo }\end{array}$ & Arecaceae & Bactris sp 2. & 20 & 6.000 & 0,106 \\
\hline 13 & Sarã de Leite & Euphorbiaceae & $\begin{array}{l}\text { Sapium obovatum Klotzsch } \\
\text { ex Müll. Arg. }\end{array}$ & 15 & 3.667 & 0,106 \\
\hline 14 & Sarã de Espinho & Ulmaceae & Celtis spinosa Spreng. $=($ C. tala $)$ & 15 & 6.667 & 0,087 \\
\hline 15 & Goiaba & Myrtaceae & Psidium guajava $\mathrm{L}$. & 20 & 8.750 & 0,072 \\
\hline 16 & Cabaça Brava & Capparaceae & Crataeva tapia L. & 25 & 8.600 & 0,070 \\
\hline 17 & Paineira & Bombacaceae & $\begin{array}{l}\text { Pseudobombax marginatum } \\
\text { (A. St. -Hil., Juss. \& Cambess.) } \\
\text { A. Robyns }\end{array}$ & 10 & 4.000 & 0,067 \\
\hline 18 & Cachuá & Meliaceae & Trichilia catigua A. Juss. & 15 & 7.333 & 0,057 \\
\hline 19 & Figueira & Moraceae & Ficus calyptroceras (Miq.) Miq. & 20 & 9.000 & 0,048 \\
\hline 20 & Pimenteira & Chrysobalanaceae & Licania parviflora Benth. & 20 & 11.000 & 0,046 \\
\hline 21 & Goiaba brava & Myrtaceae & Psidium guineense $\mathrm{Sw}$. & 10 & 7.500 & 0,045 \\
\hline 22 & Acuri & Arecaceae & $\begin{array}{l}\text { Scheelea phalerata } \\
\text { (Mart. Ex Spreng.) Burret }\end{array}$ & 10 & 9.500 & 0,038 \\
\hline 23 & Bocaiúva & Arecaceae & $\begin{array}{l}\text { Acrocomia aculeata (Jacq.) } \\
\text { Lodd.. ex Mart. }\end{array}$ & 5 & 5.000 & 0,030 \\
\hline 24 & Carandá & Arecaceae & $\begin{array}{l}\text { Copernica alba Morong } \\
\text { ex Morong \& Britoon }\end{array}$ & 5 & 6.000 & 0,029 \\
\hline 25 & Cabaça Grande & Bignoniaceae & Crescentia cujete $\mathrm{L}$. & 5 & 8.000 & 0,027 \\
\hline 26 & Ingá de Botoado & Mimosaceae & Ingá vera Willd & 5 & 7.000 & 0,020 \\
\hline 27 & Juá Vermelho & Solanaceae & Solanum varium Dunal & 5 & 10.000 & 0,013 \\
\hline 28 & Pateiro & Chrysobalanaceae & $\begin{array}{l}\text { Couepia uiti (Mart. Et Zucc.) } \\
\text { Beth. Ex Hook. f. }\end{array}$ & 5 & 7.000 & 0,013 \\
\hline 29 & Caneleira & Lauraceae & $\begin{array}{l}\text { Ocotea suaveolens (Meisn.) } \\
\text { Benth. \& Hook. f. ex Hieron. }\end{array}$ & 5 & 10.000 & 0,013 \\
\hline 30 & Manga & Anacardiaceae & Mangifera indica $\mathrm{L}$. & 5 & 10.000 & 0,005 \\
\hline
\end{tabular}

* Números em negrito na coluna do Índice de Smith demonstram as quebras ou rupturas no conhecimento dos pescadores.

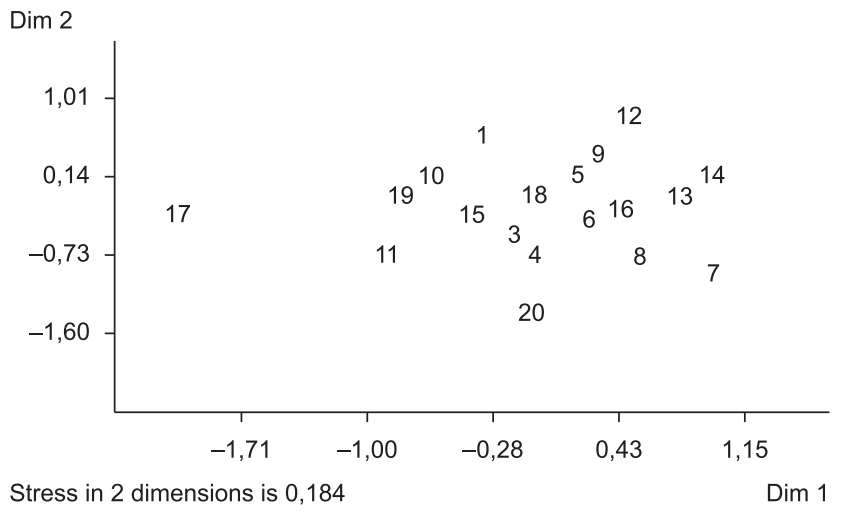

Figura 1. Diagrama de escalonamento multidimensional, apresentando os agrupamentos dos pescadores de acordo com suas respostas, Comunidade de Estirão Comprido.

Figure 1. Multidimensional scaling diagram, presenting groups of fishermen according to their responses, Community of Estirão Comprido.

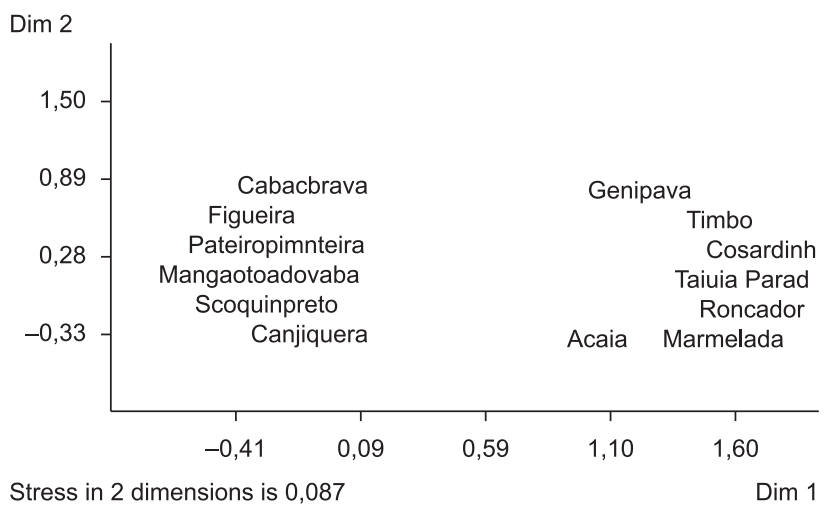

Figura 2. Diagrama de escalonamento multidimensional, apresentando os agrupamentos das fruteiras de acordo com a resposta dos informantes, Comunidade de Estirão Comprido.

Figure 2. Multidimensional scaling diagram, presenting groups of fruit trees according to the fishermen's responses, Community of Estirão Comprido. 
Tabela 2. Análise de consenso cultural sobre o conhecimento de espécies de fruteiras conhecidas para pescar pelos pescadores da Comunidade de Estirão Comprido. Table 2. Analysis of the cultural consensus on the knowledge of fruit tree species known by fishermen for fishing in the Community of Estirão Comprido.

\begin{tabular}{|c|c|c|c|c|c|c|}
\hline Fator & Valor & $\begin{array}{c}\text { Variância } \\
(\%)\end{array}$ & $\begin{array}{c}\text { Cumulativa } \\
(\%)\end{array}$ & Razão & Entrevistados & $\begin{array}{l}\text { Estimativa do } \\
\text { conhecimento }\end{array}$ \\
\hline 1 & 10,215 & 84,6 & 84,6 & 10.044 & 1 & 0,44 \\
\hline 2 & 1,017 & 8,4 & 93,0 & 1.206 & 2 & 0,63 \\
\hline 3 & 0,843 & 7,0 & 100 & - & 3 & 0,66 \\
\hline- & - & - & - & - & 4 & 0,92 \\
\hline - & - & - & - & - & 5 & 0,92 \\
\hline- & - & - & - & - & 6 & 0,85 \\
\hline - & - & - & - & - & 7 & 0,63 \\
\hline - & - & - & - & - & 8 & 0,55 \\
\hline- & - & - & - & - & 9 & 0,66 \\
\hline- & - & - & - & - & 10 & 0,72 \\
\hline- & - & - & - & - & 11 & 0,69 \\
\hline- & - & - & - & - & 12 & 0,62 \\
\hline- & - & - & - & - & 13 & 0,66 \\
\hline- & - & - & - & - & 14 & 0,75 \\
\hline- & - & - & - & - & 15 & 0,90 \\
\hline- & - & - & - & - & 16 & 0,87 \\
\hline- & - & - & - & - & 17 & 0,38 \\
\hline- & - & - & - & - & 18 & 0,72 \\
\hline- & - & - & - & - & 19 & 0,79 \\
\hline- & - & - & - & - & 20 & 0,62 \\
\hline
\end{tabular}

Tabela 3. Análise de consenso cultural do empilhamento das fruteiras pelo valor comercial do pescado na Comunidade de Estirão Comprido.

Table 3. Analysis of the cultural consensus on the piling of fruit trees according to the commercial value of the fish obtained in the Community of Estirão Comprido.

\begin{tabular}{|c|c|c|c|c|c|c|}
\hline Fator & Valor & $\begin{array}{c}\text { Variância } \\
(\%)\end{array}$ & $\begin{array}{c}\text { Cumulativa } \\
(\%)\end{array}$ & Razão & Entrevistados & $\begin{array}{l}\text { Estimativa do } \\
\text { conhecimento }\end{array}$ \\
\hline 1 & 4,525 & 83,5 & 83,5 & 10.145 & 1 & 0,72 \\
\hline 2 & 0,446 & 8,2 & 91,8 & 1.001 & 2 & 0,50 \\
\hline 3 & 0,446 & 8,2 & 100 & - & 3 & 0,62 \\
\hline- & 5,416 & 100 & - & - & 4 & 0,21 \\
\hline- & - & - & - & - & 5 & 0,71 \\
\hline- & - & - & - & - & 6 & 0,66 \\
\hline- & - & - & - & - & 7 & 0,61 \\
\hline- & - & - & - & - & 8 & 0,68 \\
\hline- & - & - & - & - & 9 & 0,77 \\
\hline- & - & - & - & - & 10 & 0,80 \\
\hline- & - & - & - & - & 11 & 0,57 \\
\hline
\end{tabular}

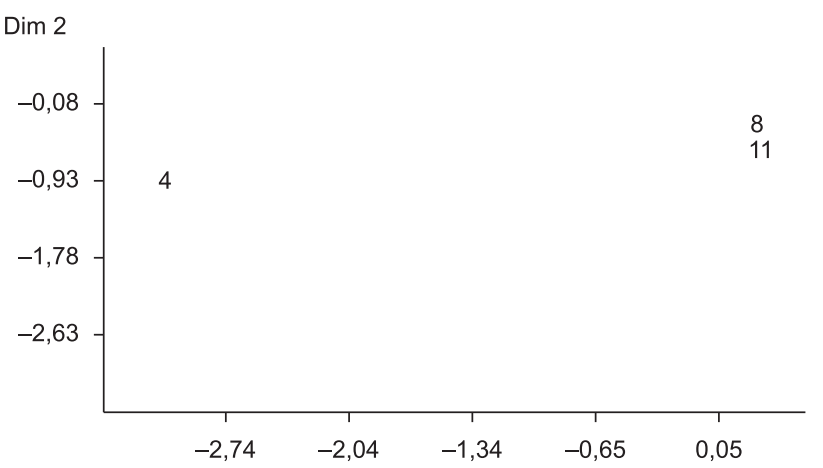

Stress in 2 dimensions is 0,003

$\operatorname{Dim} 1$

Figura 3. Diagrama de escalonamento multidimensional do empilhamento, apresentando os agrupamentos dos pescadores de acordo com suas respostas, Comunidade de Estirão Comprido.

Figure 3. Multidimensional scaling diagram of the piling, presenting groups of fishermen according to their responses, Community of Estirão Comprido.

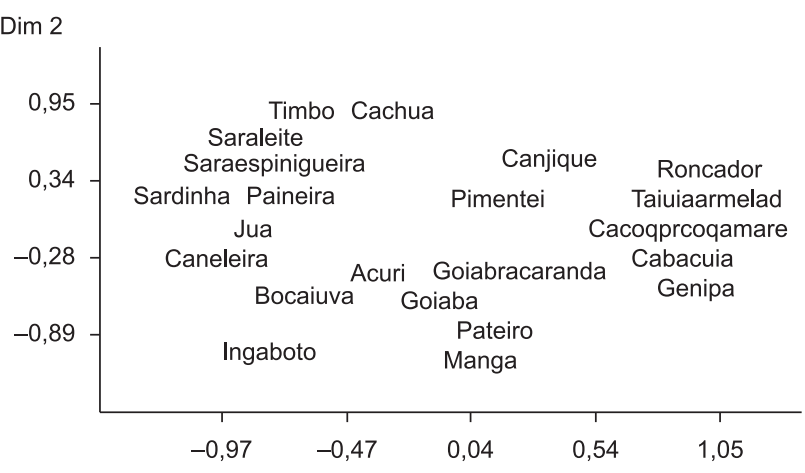

Stress in 2 dimensions is 0,108

$\operatorname{Dim} 1$

Figura 4. Diagrama de escalonamento multidimensional do empilhamento, apresentando os agrupamentos das fruteiras de acordo com valor comercial do peixe, Comunidade de Estirão Comprido.

Figure 4. Multidimensional scaling diagram of the piling, presenting the groups of fruit trees according to the commercial value of fish, Community of Estirão Comprido. 


\section{Discussão}

O domínio cultural sobre fruteiras conhecidas para pescar pelos pescadores da Comunidade de Estirão Comprido concentra-se em 30 espécies, pertencentes a 21 famílias botânicas. Das 298 espécies de plantas com informações sobre uso no Pantanal, 32 são consideradas itens utilizados por peixes na alimentação (Pott, A. \& Pott, V.J. 1994). Na Amazônia, Gottsberger (1978), estudando a dispersão de sementes por peixes em áreas de várzea alagadas, encontrou 33 espécies de plantas, metade adaptada para serem dispersas por peixes e 17 utilizadas como alimento de peixes e apreciadas como isca por pescadores. Outros estudos realizados no Pantanal e Amazônia mostram a associação de espécies frutíferas e peixes (Goulding et al. 1988, Kubitzki \& Ziburski 1994, Anjos Silva \& Da Silva 2000) evidenciando a importância desses no ambiente.

A parada $(P$. glomerata), única espécie da primeira ruptura da listagem livre, não foi observada na prática da pesca durante o período de estudo na comunidade, pois segundo alguns pescadores, seu fruto é "mole" quando maduro e sai facilmente do anzol. Porém, estudos indicam que, no Pantanal, o fruto da parada serve de alimento para peixes e é muito apreciada como isca para capturar o pacu (Piaractus mesopotamicus) (Pott, A. \& Pott, V.J. 1994) e, na Amazônia, para pescar o tambaqui, Colossoma macropomos (Maia 2001). As bordas das cordilheiras, "cordões" arenosos com altura de um a três metros acima da planície alagável, cobertos por vegetação de Cerrado, Cerradão e Mata (Junk \& Da Silva 1999), onde esta planta é abundante, tornamse local apreciado como ceva natural para pesca do pacu durante o período de enchente no Pantanal, pois o fruto cai na água e serve de alimento para os peixes. Na segunda ruptura também estão plantas frutíferas que, segundo os pescadores, são apreciadas na captura do pacu (P. mesopotamicus), piraputanga (Brycon sp.) e pacu-peva (Milossoma spp), como por exemplo, o coquinho (Bactris glaucescens), cujo uso também foi observado como isca. Estudos indicam que os frutos de B. glaucescens servem de alimento para vários animais, entre eles o pacu (Ferreira et al. 2000, Pott, A. \& Pott, V.J. 1994).

"No caso do pacu, a melhor isca que eu pesco com ele é o Coquinho, porque ele é mais duro..., você pode puxar ele não sai do anzol”. Pescador de Estirão Comprido.

Das 30 espécies da listagem livre, 22 frutificam nos períodos da enchente e cheia (Pott, A. \& Pott, V.J. 1994), favorecendo a alimentação dos peixes. Segundo Junk \& Da Silva (1999), animais aquáticos fazem migrações longitudinais e laterais, a fim de se beneficiarem durante as enchentes da produtividade das áreas alagáveis. Na Amazônia, durante a enchente, diferentes espécies frutificam, forçando os peixes a migrarem para área alagável da floresta à procura de frutos (Waldhoff \& Alencar 2000). Com as águas altas os peixes entram nas áreas alagadas, onde se alimentam de folhas jovens, frutos, sementes, insetos e outros (Maia 2001). No Pantanal, a oferta de alimentos para os peixes frugívoros é maior na época da cheia, quando as águas alcançam as plantas herbáceas, arbustos e árvores em frutificação (Da Silva \& Silva 1995).

No Pantanal, a disponibilidade de unidades de recursos para os pescadores varia no espaço e no tempo, em função da dinâmica anual das águas (Junk \& Da Silva 1999). Na enchente e cheia, as "fruteiras" constituem a unidade de recursos mais importante, distribuídas nas margens dos rios, baías e nas áreas alagáveis (Da Silva \& Silva 1995). Da mesma forma, Gottsberger (1978) descreve que frutos e sementes de várzea na Amazônia são apreciados por peixes, sem levar em conta a possível existência uma adaptação natural entre a dispersão de sementes e estes animais. No entanto, Goulding et al. (1988) consideram que pode haver uma relação mutualística entre peixes e frutos. Portanto, é importante ressaltar que o desmatamento em áreas alagadas reflete na disponibilidade de recursos alimentares para os peixes, com diminuição na quantidade e qualidade nutricional de frutos e sementes (Claro Jr. et al. 2004). Neste contexto, a conservação dos habitats dessas fruteiras é de suma importância para garantir a manutenção da pesca, principal atividade econômica atual das Comunidades Tradicionais no Rio Cuiabá.

Com o crescente reconhecimento das comunidades tradicionais em ações de conservação da biodiversidade vem sendo incentivada a realização de estudos de integração homem e ambiente. De acordo com Pasa (2007) enquanto os moradores de comunidades tradicionais detiverem autonomia de trabalho e posse de suas propriedades, os recursos naturais permanecerão explorados de acordo com as técnicas desenvolvidas ao longo de anos de convivência e sabedoria, manejando e remodelando os espaços geográficos, baseados na cultura e no saber local. No México, por exemplo, Ballesté et al. (2006) discutem o efeito das mudanças culturais no uso e manejo de duas espécies de palmeiras (Sabal yapa Wrigth ex. Becc. e Sabal mexicana Mart.) importantes na economia do povo Maya. No Canadá, Fraser et al. (2006), destacam que o CET aliado ao conhecimento biológico evolutivo, contribuiu para repovoamento de peixes nos lagos do Quebec. Na China, Davis \& Wismer (2007) discutem o potencial de inclusão de comunidades em decisões de gerenciamento de práticas florestais, focando os efeitos das práticas e manejo, bem como objetivos econômicos e sociais. Sendo assim, o CET pode ser um grande aliado para proteção de habitats e espécies econômica e culturalmente importantes, além de colaborar para o planejamento de medidas voltadas à conservação da biodiversidade nas áreas alagáveis. Para o Pantanal, uma boa estratégia seria aliar o conhecimento tradicional dos pescadores na elaboração de políticas públicas de pesca, que há anos vem carecendo de uma participação das comunidades nos processos de discussão.

Com base nas informações obtidas nesta pesquisa, foi possível verificar que os pescadores da Comunidade de Estirão Comprido detêm um amplo conhecimento acerca de fruteiras do Pantanal, bem como da sua importância na alimentação dos peixes, principalmente aqueles de alto valor comercial.

Ainda que as comunidades tradicionais no Pantanal reconheçam o papel das fruteiras na sua principal atividade econômica atual, a pesca, os efeitos da retirada da vegetação para agricultura e pecuária extensiva realizada por grandes latifundiários já se faz sentir. Além disso, o CET dessas comunidades é pouco reconhecido e valorizado, suas demandas e agendas não são atendidas pelas políticas públicas e ações de governo. Somente em 2007, o governo Federal publicou o decreto da Política Nacional de Desenvolvimento Sustentável dos Povos e Comunidades Tradicionais (Brasil 2007), que ainda não teve reflexos nas políticas estaduais de Mato Grosso, cujas práticas têm levado essas comunidades a exclusão social e ecossistêmica, empobrecendo-as e aumentando a pressão sobre recursos vegetais e pesqueiros.

\section{Agradecimentos}

Ao projeto "Avaliação das Estratégias de Pesca Utilizadas por Comunidades Tradicionais e não Tradicionais no Pantanal", da Rede de Sustentabilidade da Pesca do Centro de Pesquisa do Pantanal - CPP processo 016/04, financiado pelo Ministério da Ciência e Tecnologia (MCT), à Capes pela bolsa de mestrado concedida, à Comunidade pelo apoio e amizade durante toda a pesquisa.

\section{Referências Bibliográficas}

ANJOS SILVA, J.E. \& DA SILVA, J.C. 2000. O saber da comunidade tradicional da Baía Acurizal sobre as relações ecológicas entre as "Fruteiras" e a ictiofauna (Rio Cuiabá, Pantanal de Barão de Melgaço, MT). In III Simpósio sobre Recursos Naturais e Sócio-econômicos do Pantanal. Embrapa Pantanal, Corumbá, p.1-38. 
BALLESTLÉ, A.M., MARTORELL, C. \& CABALLERO, J. 2006. Cultural or ecologycal sustainability? The effect of cultural change on sabal palm management among the lowland Maya and Mexico. Ecol. Soc. 11(2):27.

BARRETO FILHO, H. 2006. Populações tradicionais: introdução à critica da ecologia política de uma noção. In Sociedades Caboclas Amazônicas: Modernidade e Invisibilidade (C. Adams, R.S.S. Murrieta \& W.A. Neves, Ed.). Annablume, São Paulo, p.109-143.

BEGOSSI, A. 2004. Ecologia Humana. In Ecologia de Pescadores da Mata Atlântica e da Amazônia (A. Begossi, Org.). NUPAUB-USP/HUCITEC/ FAPESP/NEPAN-UNICAMP, São Paulo, p.13-36.

BERKES, F., COLDING, J. \& FOLKE, C. 1998. Rediscovery of traditional ecological knowledge as adaptive management. Ecol. Appl. 10(5):1251-1262.

BERNARD, H.R. 2002. Research methods in anthropology: qualitative and quantitative approaches. Altamira Press, Lanham.

BERTSCH, C., VOGL, R.C. \& DA SILVA, J.C. 2006. Ethnoveterinary medicine for cattle and horses in the northern Pantanal Matogrossense, Brazil. In IV International Congress of Ethnobotany. Ege Yayinlari, Istanbul, p.233.

BORGATTI, S.P. 1996a. Anthropac 4.0. Analytic Technologies, Natick.

BORGATTI, S.P. 1996b. Anthropac 4.0 methods guide. Analytic Technologies, Natick.

BORTOLOTTO, I.M. \& GUARIM NETO, G. 2005. O uso do camalote, Eichhornia crassipes (Mart.) Solms, Pontederiaceae e para confecção de artesanato no Distrito de Albuquerque, Corumbá, MS, Brasil. Acta Bot. Bras. 19(2):331-337.

BRASIL. 2007. Decreto n ${ }^{\circ} 6.040$. Institui a Política Nacional de desenvolvimento Sustentável dos Povos e Comunidades Tradicionais. Diário Oficial [da] República Federativa do Brasil, Brasília, DF, 08 fev. 2007.

BRASIL. Ministério das Minas e Energia. Secretaria-Geral. 1982. Geologia, Geomorfologia, Pedologia, Vegetação e uso Potencial da Terra. Projeto Radambrasil. Folha SE-21, Corumbá. Secretaria Geral, Rio de Janeiro, 448p. Levantamento de Recursos Naturais, 27.

CAMPOS FILHO, S.V.L. 2002. Tradição e ruptura: cultura e ambiente pantaneiros. Entrelinhas, Cuiabá.

CAULKINS, D. \& HYATT, S.B. 1999. Using consensus analysis to measure cultural diversity in organizations and social movements. Field Meth. 11(1):5-26

CLARO Jr., L., FERREIRA, E., ZUANON, J. \& ARAÚJO-LIMA, C. 2004 $O$ efeito da floresta alagada na alimentação de três espécies de peixes onívoros em lagos de várzea da Amazônia Central, Brasil. Acta Amaz. 34(1):133-137.

DA SILVA, C.J. \& SILVA, J.A.F. 1995. No ritmo das águas do Pantanal NUPAUB, São Paulo.

DA SILVA, C.J., WANTZEN, M., CUNHA, C.N. \& MACHADO, F.A. 2001 Biodiversity in the Pantanal Wetland, Brazil. In Biodiversity in wetlands: assessment, function and conservation (J.J. Wolfgang, B. Gopal \& J.A. Davis, Org.). Backhuys Publishers, Leiden, p.187-215.

DAVIS, E.G.R., WISMER, S.K. 2007. Sustainable Forestry and local people: the case of Hainan's Li Minority. Human Ecology. 35:415-426.

ELLEN, R. 2006. Introduction. J. Royal Anthropol. Inst. 12(S1):S1-S22.

FERREIRA, A.R., MACEDO, M. \& DA SILVA, C.J. 2000. Algumas interações bióticas de uma população de Bactris glaucescens Drude (Arecaceae/Palmae) em uma mata inundável do Pantanal de Barão de Melgaço, Mato Grosso, Brasil. In III Simpósio sobre Recursos Naturais e Sócio-econômicos do Pantanal. Embrapa, Corumbá.

FIGUEIREDO, D.M. \& DA SILVA, C.J. 1999. Caracterização limnológica do sistema de baías Chacororé - Sinhá Mariana. Rev. Mt. Geo. 3:57-75.

FRASER, D.J., COON, T., PRINCE, M.R., DION, R. \& BERNATCHEZ, L. 2006. Integrating traditional and evolutionary knowledge in biodiversity conservation: a population level case study. Ecol. Soc. 11(2):4.

GALDINO, Y.S.N. \& DA SILVA, C.J. 2007. A casa pantaneira - moradia tradicional de uma comunidade ribeirinha do Pantanal Matogrossense. In IV Encontro Nacional e II Encontro Latino Americano Sobre Edificações e Comunidades Sustentáveis. ANTAC, Campo Grande, p.1276-1285.
GALETTI, M., DONATTI, C.I., PIZO, M.A. \& GIACOMINI, H.C. 2007. Big Fish are the Best: Seed Dispersal of Bactris glaucescens by the Pacu Fish (Piaractus mesopotamicus) in the Pantanal, Brazil. Biotrópica. 40:386-389.

GOTTSBERGER, G. 1978. Seed dispersal by fish in the inundated regions of Humaitá, Amazonia. Biotrópica 10(3):170-183.

GOULDING, M., CARVALHO, M.L. \& FERREIRA, E.G. 1988. Rio Negro: rich life in poor water: Amazonian diversity and foodchain ecology as seen through fish communities. SPB Academic Publishing, The Hague.

GUARIM NETO, G. \& CARNIELLO, M.A. 2007. Etnoconhecimento e saber local: um olhar sobre populações humanas e os recursos vegetais. In Povos e paisagens: etnobiologia, etnoecologia e biodiversidade no Brasil (U.P. Albuquerque, C.G.A. Alves \& T.A.S. Araújo, Org.). NUPEEA/UFRPE, Recife, p.105-114.

GUARIM NETO, G. 2006. O saber tradicional Pantaneiro: as plantas medicinais e a educação ambiental. Rev. Eletrônica Mestr. Educ. Ambient. 17:71-89.

HANAZAKI, N. 2004. Etnobotânica. In Ecologia de Pescadores da Mata Atlântica e da Amazônia (A. Begossi, Org.). HUCITEC/NEPAMUNICAMP/NUPAUB-USP, São Paulo, p.37-57.

IBGE. 2001. Populações e Domicílios. Censo 2000 com Divisão Territorial 2001. http://www.ibge.gov.br/cidadesat/default.php (último acesso em 29/01/2002).

JUNK, W.J. \& DA SILVA, C.J. 1999. O conceito de Pulso de Inundação e suas implicações para o Pantanal de Mato Grosso. In II Simpósio Sobre Recursos Naturais e Sócio-econômicos do Pantanal. Embrapa, Corumbá, p.17-28.

KUBITZKI, K. \& ZIBURSKI, A. 1994. Seed dispersal in flood plain forests of Amazonia. Biotrópica 26(1):30-43.

MAIA, L.M.A. 2001. Frutos da Amazônia: fonte de alimentos para peixes. Programa de Desenvolvimento Empresarial e Tecnológico. SEBRAE/ AM, Manaus.

MORAIS, F.F., MORAIS, R.F. \& DA SILVA, C.J. 2009. Conhecimento ecológico tradicional sobre plantas cultivadas pelos pescadores da comunidade Estirão Comprido, Pantanal Matogrossense, Brasil. Bol. Mus. Pará Emílio Goeldi Cienc. Hum. 4(2):277-294.

PASA, M.C. 2007. Um olhar etnobotânico sobre as comunidades do BambaMT. Entrelinhas, Cuiabá.

PASA, M.C., SOARES, J.J. \& GUARIM NETO, G. 2005. Estudo etnobotânico na comunidade de Conceição-Açu (alto da bacia do rio Aricá Açu, MT, Brasil). Acta Bot. Bras. 19(2):195-207.

POTT, A. \& POTT, V.J. 1994. Plantas do Pantanal. Embrapa-CPAP, Corumbá.

PURI, R.K. 2001. Anthropac for environment and anthropology, introduction to anthropac for environment and anthropology. http://www.uka.ac.uk/ anthropology/staff/rajP.html

REYS, A.P., SABINOB, J. \& GALETTI, M. 2009. Frugivory by the fish Brycon hilarii (Characidae) in western Brazil. Acta Oecol. 35:136-141.

SCHWENK, L.M. \& DA SILVA, C.J. 1999. Uso da terra e vegetação na região de Mimoso no Pantanal Mato-Grossense. Rev. Mat. Geo. 3(4):121-150.

WALDHOFF, D. \& ALENCAR, M.L. 2000. Production and chemical composition of fruit from trees in floodplain forests of central Amazonia and their importance for fish production. In The central Amazon floodplain: actual use and options for a sustainable management. (W.J. Junk, J.J. Ohly, M.T.F. Piedade \& M.G.M. Soares, Ed.). Backhuys Publishers, Leiden, p.393-41.

WELLER, S.C. \& ROMNEY, A.K. 1988. Systematic Data Collection. Sage Publications, California.

ZUNTINI, D., VICENTIN, W., COSTA, F.E.S., MARQUES, S.P. \& BARBOZA, E.G. 2004. Alimentação natural da piraputanga, Brycon hilarii (Teleostei - Characidae) no Rio Miranda, Município de Jardim, MS - Projeto Piracema. In IV Simpósio sobre Recursos Naturais e Sócioeconômicos do Pantanal. Embrapa Pantanal, Corumbá. 